\title{
Hemodynamic rounds: Ascending aortic dissection as a mechanism of hemodynamic instability
}

\author{
Stéphane Coutu, MD • Alain Deschamps, MD, PhD • \\ André Denault, MD, PhD
}

Received: 23 March 2010/Accepted: 30 July 2010/Published online: 17 August 2010

(C) Canadian Anesthesiologists' Society 2010

The Research Ethics Boards of the Montreal Heart Institute and the Centre Hospitalier Universitaire de Sherbrooke granted approval to use the echocardiographic images for research purposes. Consent for publication of this report was obtained from a family member.

Acute aortic dissection of the ascending aorta is potentially a highly lethal condition requiring rapid diagnosis and urgent surgical treatment. Since its introduction to clinical practice, transesophageal echocardiography (TEE) has become an invaluable tool in guiding the diagnosis and repair of ascending aortic dissection. A specific echographic sign for aortic dissection is an intraluminal mobile thin membrane that separates a true from a false lumen and shows systolic convexity towards the false lumen. Compared with magnetic resonance imaging and angioscanning, TEE has a high sensitivity (99-100\%) and specificity (68$97 \%$ ) for aortic dissection. ${ }^{1,2}$ Recent practice guidelines for perioperative TEE specify that TEE should be used in all open heart and thoracic aortic surgeries. ${ }^{3}$ However, sometimes TEE may fail to detect distal ascending dissection

Electronic supplementary material The online version of this article (doi:10.1007/s12630-010-9370-9) contains supplementary material, which is available to authorized users.

S. Coutu, MD

Department of Anesthesiology, Centre Hospitalier Universitaire de Sherbrooke, Sherbrooke, QC, Canada

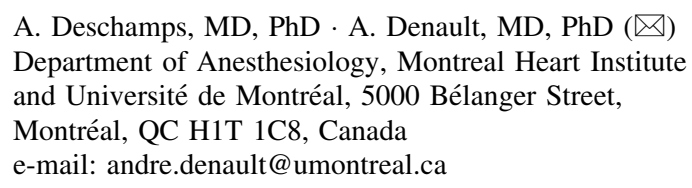

A. Denault, $\mathrm{MD}, \mathrm{PhD}$

Division of Critical Care, Centre Hospitalier de l'Université de Montréal, Montréal, QC, Canada because of the interposition of large airways between the aorta and the esophagus, the so-called blind spot. Direct epiaortic ultrasound scanning can overcome this limitation and thus facilitate decision-making for the surgical treatment to follow. ${ }^{4}$ Furthermore, linear artefacts, reverberation, and side lobes may also mislead the clinician to a false diagnosis of aortic dissection.

Transesophageal echocardiography encompasses many critical roles in the management of aortic dissection (Table 1), and it is a very useful tool in assessing the etiology of hemodynamic instability (Table 2). Bossone et $a .^{2}$ have even shown a prognostic role of TEE by revealing a higher mortality in acute Type A aortic dissection-in patients with epicardial effusion, tamponade, periaortic hematoma, and patent false lumen-compared. The role of TEE in aortic dissection is illustrated in the following case.

A 66-yr-old woman presented to the emergency room for right facial numbness and tearing sternal pain. An angioscan showed a complex Type A aortic dissection (Fig. 1). The patient was then rushed to the operating room. At this time, the patient was hemodynamically stable with no signs of hypoperfusion aside from her right-sided neurological symptoms. During echoguided right jugular central venous catheterization, dissection with complete obstruction of the right carotid artery was noted (Fig. 2, Video 1a), but normal flow was seen in the left carotid artery (Video $1 b$ ). The TEE exam showed a pericardial effusion with right diastolic atrial compression (Fig. 3, Video 2), normal right and left contractility, trace aortic insufficiency (AI), and proximal aortic dissection extending into the descending thoracic aorta.

The surgeon proceeded to femoral arterial cannulation in order to institute cardiopulmonary bypass (CPB). For a 19-min period, the patient was subjected to deep 
Table 1 Roles of transesophageal echocardiography in aortic dissection

1. Diagnosis of aortic dissection

2. Diagnosis of complications of aortic dissection:

Myocardial ischemia

Aortic insufficiency

Pericardial and thoracic effusion

3. Identification of the true and false lumens

4. Assessment of the branches of the aortic arch

5. Identification of the entry tears ( \pm epiaortic scanning)

6. Evaluation of heart valves

7. Evaluation of placement of aortic and venous cannulae

8. Complete post-cardiopulmonary bypass assessment

Table 2 Etiologies of hemodynamic instability in aortic dissection

Reduction in mean systemic venous pressure

1. Thoracic or abdominal aortic rupture

2. Vasodilatory shock if prolonged shock and acidosis

Increases in right atrial pressure

1. Cardiac ischemia (coronary dissection)

2. Aortic insufficiency from:

Aortic valve involvement in dissection

Intimal flap prolapsing in the aortic valve

Aortic root dilatation

3. Mitral valve insufficiency (prolapse in Marfan syndrome or restriction from ischemia)

4. Right pulmonary artery compression by aortic dilatation

5. Cerebral ischemia and brain-heart syndrome

Resistance to venous return

1. Cardiac tamponade

2. Tension hemothorax

3. Abdominal compartment syndrome

hypothermic circulatory arrest for the distal anastomosis with a Hemashield graft 28 (Maquet, Cardiovascular LLC, Wayne, NJ, USA). A suspect innominate dissection and occlusion from the surface echography (right carotid occlusion) excluded the possibility of axillary cannulation and antegrade cerebroprotection. Cardiopulmonary bypass was then reinstituted, and the proximal anastomosis was performed at the sinotubular junction with preservation of the aortic valve.

Transesophageal echocardiography post-bypass showed normal right and left contractility and a trace of aortic insufficiency. The next day, the patient needed further surgery for bleeding. She also suffered acute lung injury syndrome, and several days were necessary to wean her from the ventilator. During that time, the patient required sodium nitroprusside and labetolol to control her high blood pressure. A week later, just before she was

\section{Aortic intimal flap}

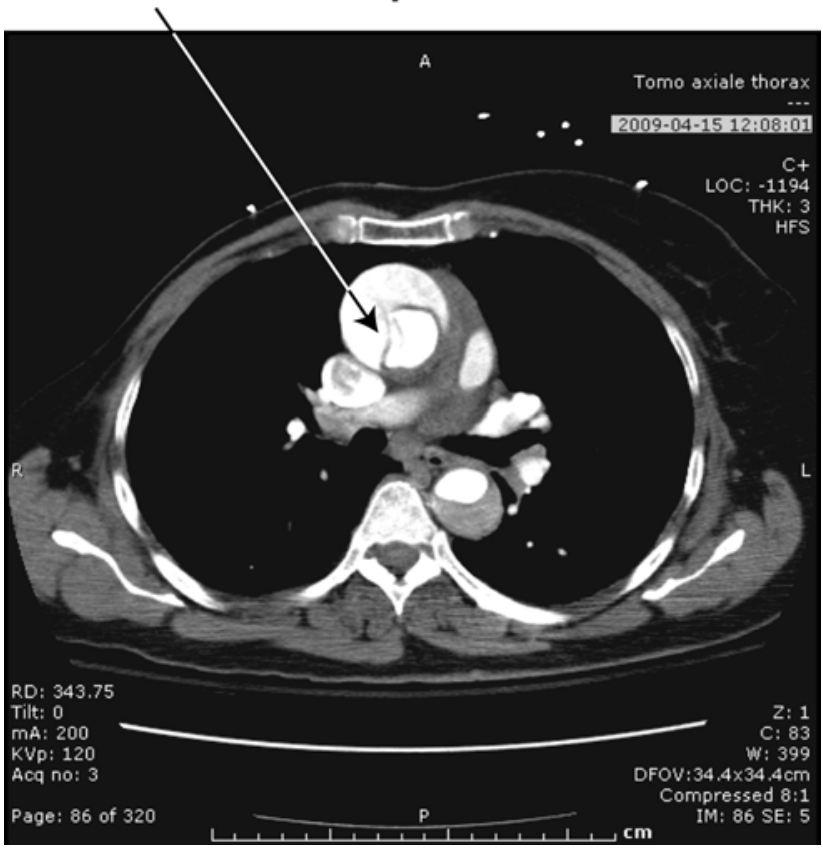

Fig. 1 Computed tomographic scan showing the intimal flap diagnostic of aortic dissection

transferred from the intensive care unit to the ward, the patient coughed and suddenly collapsed. Cardiopulmonary resuscitation was undertaken, and during chest compression, a TEE examination showed extrinsic left atrial compression (Fig. 4, Video 3) and a massive thoracic left hemothorax (Fig. 5, Video 4). A ruptured descending aorta was raised in the differential diagnosis. In the operating room, no blood was found in the pericardial space, but a massive left hemothorax was observed and partially aspirated. Following chest tube drainage, the patient's hemodynamic condition stabilized and vasopressors were weaned. At that point, based on the TEE findings of a probable thoracic origin of the rupture site (Fig. 6), a decision was taken to insert an endograft in the descending thoracic aorta. Aortic stenting, (Zenith, Cookmedical, Bloomington, IN, USA) was performed in the interventional radiology suite. The procedure was difficult, and after stenting the descending aorta, a re-entry leak was found around the distal anastomosis near the innominate trunk between the true and the false lumen. Stenting of the ascending aorta was performed. At the end of the procedure, another stent needed to be placed to close an endoleak (Type 1) in the proximal end of the descending aortic stent. Unfortunately, this resulted in an iatrogenic occlusion of the left subclavian artery. Blood flow was still present in the innominate trunk and the left carotid artery. Following the procedure, the patient suffered a massive stroke in the left sylvian territory. The etiology of the massive stroke was unclear. Because of the patient's poor 
A

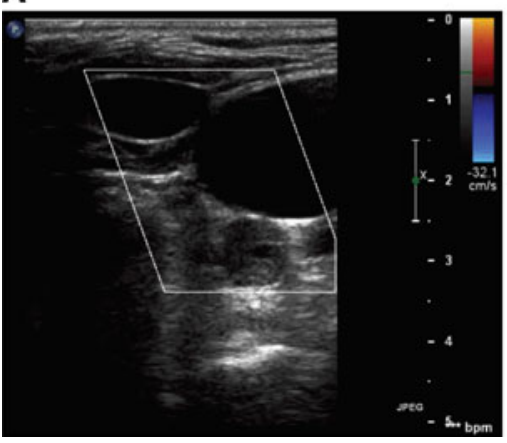

B

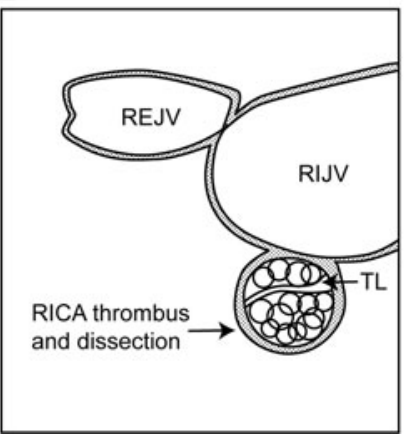

C

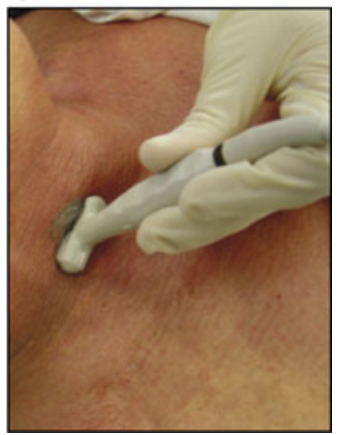

Fig. 2 (A-B) Short-axis transverse view at the level of the right internal jugular vein (RIJV). Dissection and thrombosis of the right internal carotid artery (RICA) is present. (C) An example is shown of the position of the ultrasound probe to obtain this view. REJV = right external jugular vein; $\mathrm{TL}=$ true lumen. Video 1a showing absence of colour Doppler flow in the right common carotid artery and Video $1 \mathrm{~b}$ of the same patient showing normal colour Doppler flow in the left common carotid artery (with permission from Denault et al.). ${ }^{5}$
Fig. 3 (A-B) Upper to midesophageal view at 0 showing the dilatated aorta (Ao) with the false lumen (FL), the associated pericardial effusion (PE) with thrombus, and the reduced dimension of the right atrium (RA). LA = left atrium; RVOT $=$ right ventricular outflow tract; $\mathrm{TL}=$ true lumen (see Video 2)

Fig. 4 (A-B) Mid-esophageal four-chamber view with external compression behind the left atrium (LA) most likely resulting from the left hemothorax. This region corresponds with the oblique sinus. $\mathrm{LV}=$ left ventricle; $\mathrm{RA}=$ right atrium; $\mathrm{RV}=$ right ventricle (see Video 3)

Fig. 5 (A-B) Mid-esophageal long-axis view of the descending thoracic aorta (Ao) with left-sided rotation showing a massive left pleural hemothorax (see Video 4)
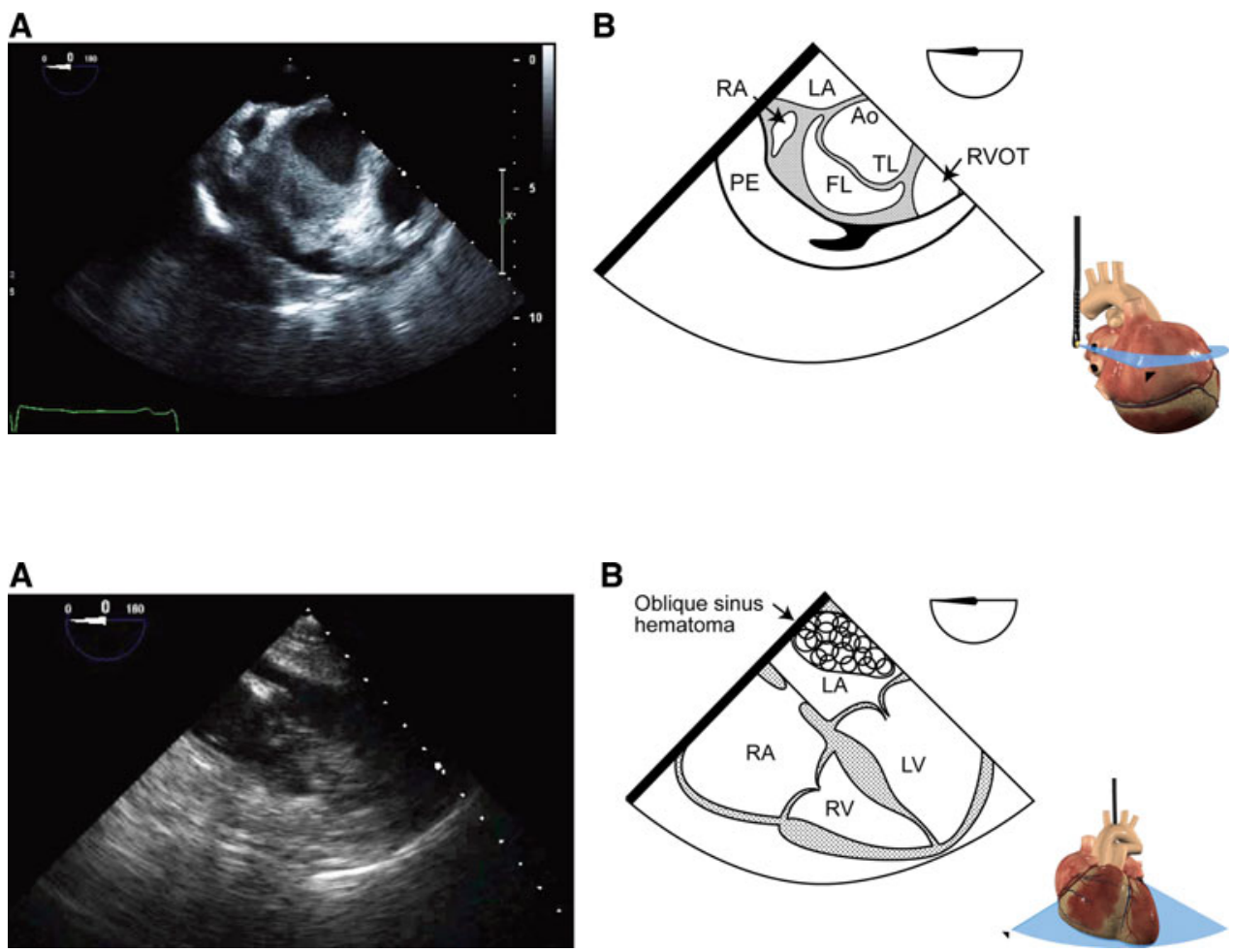

B
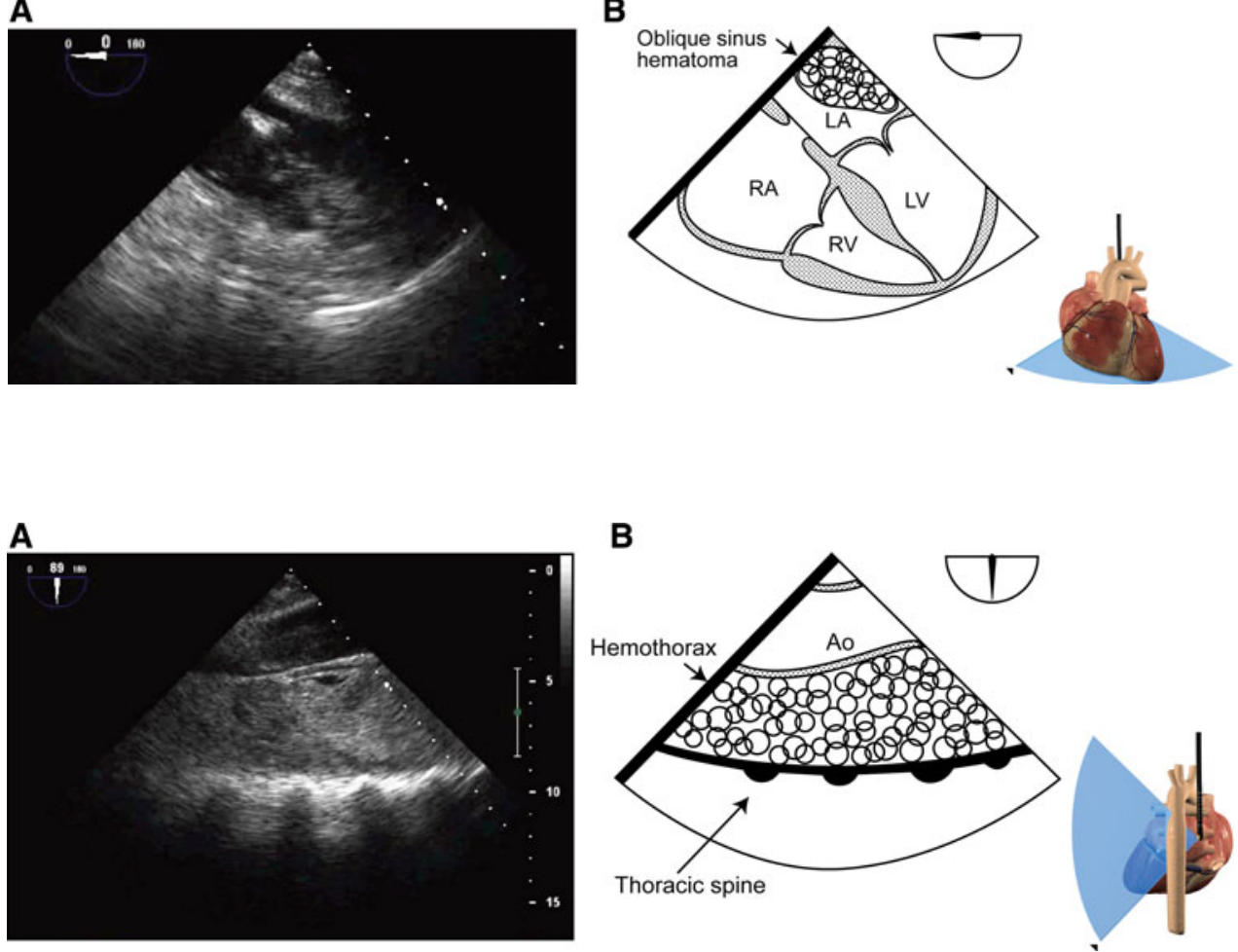
Fig. 6 Descending aortic longaxis view of the descending aorta showing a 7.3-mm aortic wall thickening diagnostic of an aortic hematoma. Aortic wall rupture at the site of the hematoma is the most probable etiology of the massive left hemothorax. Ao = aorta; $\mathrm{FL}=$ false lumen; $\mathrm{TL}=$ true lumen
A

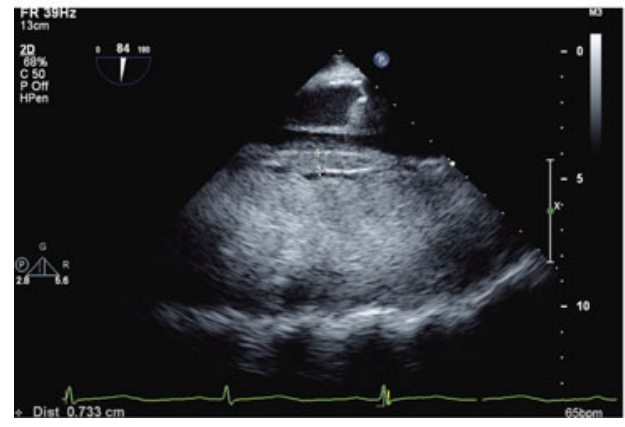

B

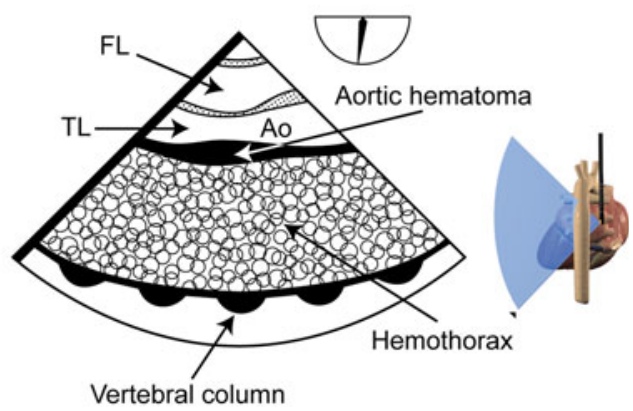

prognosis, the family consulted with the treating physicians and decided to withdraw further medical support. The patient died very soon thereafter.

\section{Discussion}

Patients with acute aortic dissection normally describe sudden severe chest pain irradiating to the back. Risk factors include hypertension, previous aortic surgeries, and congenital syndromes (Marfan's, Turner's). ${ }^{5}$ Ascending aortic dissection is a surgical emergency.

Transesophageal echocardiography has now become a critical monitoring tool in the assessment of hemodynamic instability in aortic dissection. Hemodynamic instability in aortic dissection can result from numerous factors, which are summarized in Table 2. The mechanism of hemodynamic instability can be approached using Guyton's concept of venous return combined with bedside echography. ${ }^{6}$ In this concept, venous return and cardiac output depend on three variables: the mean systemic filling pressure (Pms), the right atrial pressure (Pra), and the resistance to venous return (Rvr). Venous return is proportional to the difference between Pms and Pra and inversely proportional to Rvr.

First, Pms can fall from massive hemorrhage, and vasodilatory shock can result if the shock state is prolonged. ${ }^{7}$ Right atrial pressure can increase if coronary artery perfusion is compromised from ostial dissection. This condition can lead to either left or right ventricular systolic or diastolic dysfunction with or without mitral regurgitation. Acute aortic insufficiency from aortic dilation of leaflet disruption, will also lead to an increase in Pra as a result of several mechanisms. Right atrial pressure can also become elevated from right coronary ischemia through dissection or compression, and in some cases, the aortic false lumen can compress the right pulmonary artery. Both conditions will result in acute right ventricular failure.

Resistance to venous return will be impeded as in this case with pericardial tamponade and hemothorax. Abdominal hypertension leading to abdominal compartment syndrome from free abdominal or retroperitoneal hemorrhage will also increase Rvr. ${ }^{8}$ Atrial cavities will be compressed in the case of tamponade and massive thoracic hemorrhage, as shown in this patient. Several of these conditions can be diagnosed using TEE at the bedside. ${ }^{5}$

In addition, TEE can be used to evaluate flow in the thoracic and abdominal aortic branches ${ }^{5}$ and could be considered as an indirect monitor of cerebral blood flow by measuring carotid flow, especially of the left carotid artery.

Epiaortic scanning can also be used in assessing perfusion of the right innominate artery and in looking for a re-entry site in the TEE blind spot. As showed in this case, surface echography can also be very beneficial for planning the surgery. Epicardial echocardiography can facilitate aortic cannulation, and femoral surface echography will identify the true arterial lumen for invasive blood pressure monitoring.

Trained anesthesiologists and critical physicians should consider using TEE in all tracheally intubated patients suspected of aortic dissection. A complete TEE exam is also essential for assessing the etiology of hemodynamic instability in these patients (like thoracic tamponade as shown in this case). Other echocardiographic modalities, such as surface echography and epiaortic echography, can also be very important in decision-making for this type of pathology.

In conclusion acute aortic dissection is a highly lethal condition. Despite the unfortunate outcome in this case, the complexity of the hemodynamic course, its ongoing evaluation, and the assessment of responses to intervention show how careful monitoring with TEE can guide clinicians regarding clinical decision-making and the surgical management of this condition.

Competing interests None declared.

\section{References}

1. Nienaber CA, Spielmann RP, von Kodolitsch $Y$, et al. Diagnosis of thoracic aortic dissection. Magnetic resonance imaging versus transesophageal echocardiography. Circulation 1992; 85: 434-47. 
2. Bossone E, Evangelista A, Isselbacher E, International Registry of Acute Aortic Dissection Investigators, et al. Prognostic role of transesophageal echocardiography in acute type A aortic dissection. Am Heart J 2007; 153: 1013-20.

3. American Society of Anesthesiologists and Society of Cardiovascular Anesthesiologists Task Force on Transesophageal Echocardiography. Practice guidelines for perioperative transesophageal echocardiography. An updated report by the American Society of Anesthesiologists and the Society of Cardiovascular Anesthesiologists Task Force on Transesophageal Echocardiography. Anesthesiology 2010; 112: 1084-96.

4. Demertzis S, Casso G, Torre T, Siclari F. Direct epiaortic ultrasound scanning for the rapid confirmation of intraoperative aortic dissection. Interact Cardiovasc Thorac Surg 2008; 7: 725-6.
5. Denault AY, Couture P, Tardif JC, Buithieu J. Transesophageal Echocardiography Multimedia Manual: A Perioperative Transdisciplinary Approach. Oxford: Marcel Dekker; 2005.

6. Guyton AC, Lindsey AW, Abernathy B, Richardson T. Venous return at various right atrial pressures and the normal venous return curve. Am J Physiol 1957; 189: 609-15.

7. Landry $D W$, Oliver JA. The pathogenesis of vasodilatory shock. N Engl J Med 2001; 345: 588-95.

8. Deslauriers $N$, Dery $R$, Denault A. Acute abdominal compartment syndrome. Can J Anesth 2009; 56: 678-82. 